\title{
Influence of krypton atoms on the structure of hydrogenated amorphous carbon deposited by plasma enhanced chemical vapor deposition
}

\author{
M. H. Oliveira, Jr.., ${ }^{1,2, a)}$ G. A. Viana, ${ }^{1}$ M. M. de Lima, Jr., ${ }^{2}$ A. Cros, ${ }^{2}$ A. Cantarero, ${ }^{2}$ and \\ F. C. Marques ${ }^{1, b)}$ \\ ${ }^{1}$ Instituto de Física Gleb Wataghin, Universidade Estadual de Campinas-UNICAMP, C. P. 6165, Campinas, \\ São Paulo 13083-970, Brazil \\ ${ }^{2}$ Institut de Ciència dels Materials, Universitat de València, E-46071 València, Spain
}

(Received 7 June 2010; accepted 16 November 2010; published online 30 December 2010)

\begin{abstract}
Hydrogenated amorphous carbon $(\mathrm{a}-\mathrm{C}: \mathrm{H})$ films were prepared by plasma enhanced chemical vapor deposition using methane $\left(\mathrm{CH}_{4}\right)$ plus krypton $(\mathrm{Kr})$ mixed atmosphere. The depositions were performed as function of the bias voltage and krypton partial pressure. The goal of this work was to study the influence of krypton gas on the physical properties of a-C:H films deposited on the cathode electrode. Krypton concentration up to 1.6 at. \%, determined by Rutherford Back-Scattering, was obtained at high $\mathrm{Kr}$ partial pressure and bias of $-120 \mathrm{~V}$. The structure of the films was analyzed by means of optical transmission spectroscopy, multi-wavelength Raman scattering and Fourier Transform Infrared spectroscopy. It was verified that the structure of the films remains unchanged up to a concentration of $\mathrm{Kr}$ of about 1.0 at. \%. A slight graphitization of the films occurs for higher concentration. The observed variation in the film structure, optical band gap, stress, and hydrogen concentration were associated mainly with the subplantation process of hydrocarbons radicals, rather than the krypton ion energy. (C) 2010 American Institute of Physics.
\end{abstract} [doi:10.1063/1.3526000]

\section{INTRODUCTION}

Amorphous carbon $(\mathrm{a}-\mathrm{C})$ structures have been extensively investigated during the last two decades. Several works have focused on the relation between the material physical properties and the different amounts and distribution of $s p^{2}$ and $s p^{3}$ hybridized carbon into the matrix. ${ }^{1-10}$ Other researches focused on how to produce a matrix with some specific characteristic, usually for a well defined application, such as high hardness, chemical inertness, low friction coefficient and low electron affinity. ${ }^{11-16}$ Today, it is well known which technique is appropriate to produce amorphous carbon films with some specific characteristic. For instance, nonhydrogenated graphiticlike carbon (GLC) is usually prepared by sputtering ${ }^{17}$ and hydrogenated GLC by plasma enhanced chemical vapor deposition (PECVD) at high self-bias. ${ }^{14,18,19}$ Diamondlike carbon (DLC) can be prepared by sputtering and its hydrogenated counterpart by PECVD with intermediate self-bias. ${ }^{14,19}$ Polymericlike carbon (PLC) is easily prepared by PECVD at very low self-bias. ${ }^{19}$ The hardest known intrinsic a-C, the so called tetrahedral amorphous carbon (ta-C), is commonly deposited by filtered cathodic vacuum arc (FCVA) ${ }^{20-24}$ mass selected ion beam, ${ }^{25}$ or pulsed laser deposition. ${ }^{26-28}$

Investigation concerning the incorporation of noble gases in carbon structures have many purposes, such as to understand the origin of noble gases in meteorites ${ }^{29,30}$ or to understand the mechanisms behind the changes of the properties (stress, friction coefficient, hydrofobicity, etc.) of

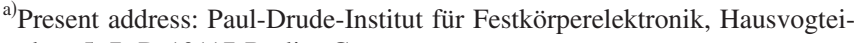
platz 5-7, D-10117 Berlin, Germany.

b) Electronic mail: marques@ifi.unicamp.br.
}

amorphous carbon films. Among these procedures, the insertion of noble gases into amorphous carbon have been investigated either through the bombardment of the film by noble gases, using an ion gun, or introducing noble gases in the atmosphere during deposition by PECVD. Pure amorphous carbon prepared by sputtering, using only argon in the atmosphere, has properties that vary from GLC to DLC. Amorphous carbon deposited by ion beam assisted deposition (IBAD), in which an ion gun is used to sputter a graphite target, while a secondary ion gun is used to simultaneously assist the deposition with a beam of noble gases $(\mathrm{Ne}, \mathrm{Ar}, \mathrm{Kr}$, and $\mathrm{Xe}$ ) with energies up to $1000 \mathrm{eV}$, are highly $s p^{2}$-hybridized, but with very high compressive stress, reaching values up to $10 \mathrm{GPa}^{17,31}$ This level of stress is typically found in highly $s p^{3}$-hybridized structures. Cheah et al. ${ }^{32} \mathrm{ob}-$ served that the concentration of $s p^{2}$ bonds in a-C, prepared by FCVA, increases by concurrent Ar ion bombardment during deposition. The increase in $s p^{2}$ sites is followed by a decrease of the optical band gap and intrinsic stress. An interesting result with the introduction of noble gases in a-C matrix was obtained by Kim et al. ${ }^{33}$ who showed that it is possible to reduce the intrinsic stress of ta-C films by about $35 \%$ without altering the $s p^{3}$ concentration by depositing the films by FCVA in argon atmosphere. This result is important because the typically high mechanical stress of ta-C films is the main factor that limits its technological applications.

Amorphous carbon deposited on the anode electrode of a PECVD system (usually a grounded electrode) is typically polymeric, transparent, very soft and have very low stress. On the other hand, films deposited on the cathode electrode (which is the negatively powered electrode) are graphiticlike or diamondlike. The last one is very hard, stressed and has 
low band gap. These effects have been attributed to the subplantation process of energetic ions, ${ }^{34-37}$ which is more efficient in the 100-200 eV range.

In this work we analyze the influence of concurrent $\mathrm{Kr}$ bombardment of amorphous carbon films during deposition using a $13.56 \mathrm{MHz}$ rf-PECVD system and depositing the films on the powered cathode electrode. For that purpose, $\mathrm{CH}_{4} / \mathrm{Kr}$ mixture was used and the bias voltage was varied in the $-30-(-550 \mathrm{~V})$ range to mimic the effect observed in films deposited by IBAD concerning the $\mathrm{Kr}$ ion energy. Structural, optical and mechanical properties of the films were investigated by optical transmission spectroscopy, Fourier transform infrared spectroscopy (FTIR), Rutherford back-scattering (RBS), intrinsic stress measurements, visible, and ultraviolet (UV) Raman spectroscopy.

\section{EXPERIMENTAL DETAILS}

Three series of amorphous carbon films were prepared by rf-PECVD. The first series of films was deposited in an atmosphere of $10^{-2}$ Torr pure methane $\left(\mathrm{CH}_{4}\right)$, as a function of the bias voltage, which varied in the $-30-(-550 \mathrm{~V})$ range. The second series of films was deposited in the same range of bias voltage using a composed atmosphere of methane (partial pressure of $7 \times 10^{-3}$ Torr) and krypton (partial pressure of $3 \times 10^{-3}$ Torr), keeping the total pressure constant at $10^{-2}$ Torr. The third series was deposited at a bias voltage of $-120 \mathrm{~V}$, which is the condition in which the incorporation of krypton atoms is higher and the films are harder. In this last series of films the krypton partial pressure was varied in the range of $0.5 \times 10^{-3}$ to $5.0 \times 10^{-3}$ Torr, keeping the total pressure constant. The system chamber was pumped down to approximately $10^{-6}$ Torr prior deposition. All samples were prepared at room temperature, using a Dressler rf-power generator model Cesar 1310. The films were deposited on silicon $\langle 111\rangle$ substrates for stress, FTIR and Raman measurements, and on corning glass 7059 substrates for optical characterization.

Optical properties were obtained by transmission spectroscopy using a Perkin Elmer spectrophotometer model Lambda 9, and the optical constants were extracted from the experimental data by means of the PUMA software. ${ }^{38-40}$ Structural information was obtained by FTIR, RBS, and visible and ultraviolet Raman spectroscopy. Infrared transmission spectra were obtained by a NICOLET FTIR spectrometer from samples deposited on silicon substrate, in the wave number ranging from 400 to $4000 \mathrm{~cm}^{-1}$, with a resolution of $4 \mathrm{~cm}^{-1}$. Each spectrum is an average of 100 scans. The transmission spectra presented interference fringes, which were eliminated by fitting the corresponding background, outside the absorption band, according to the expression: $T_{0}(v)=\tau\left[(1-\rho)^{2}+4 \rho \sin ^{2}(2 \pi n d v)\right]^{-1}$, where $d$ is the thin film thickness, $\tau$ and $\rho$ depend on the refractive index of the film and substrate, and $n$ is the refractive index of the film. The absorption coefficient $\alpha(v)$ was obtained from the LambertBeer's law. $^{41}$

Raman spectra were collected at room temperature with excitation radiation in the visible and ultraviolet ranges. Both wavelengths, 514.5 and $275.4 \mathrm{~nm}$, are emission lines from
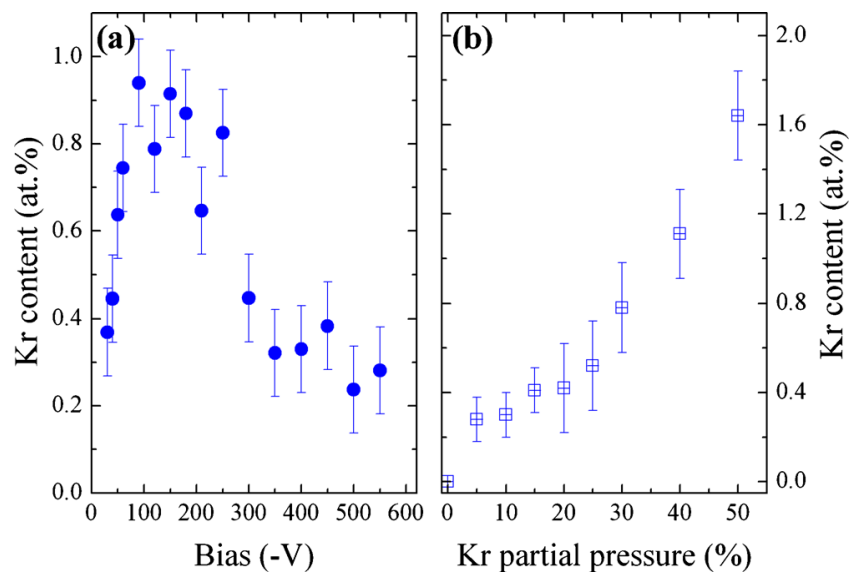

FIG. 1. (Color online) Concentration of $\mathrm{Kr}$ atoms incorporated into a-C:H films deposited by PECVD as a function of the (a) bias voltage and (b) $\mathrm{Kr}$ partial pressure.

Argon laser. In the UV range a prism monochromator was used to avoid the incidence of Argon laser plasma lines on the sample, which are in the same range of the carbon Raman shift. The spectral resolution was about $1 \mathrm{~cm}^{-1}$ with a scattering configuration close to the backscattering geometry. A $100 \times$ objective was employed to focus the visible light and $40 \times$ for the ultraviolet. The experiments were performed using an HORIBA Jobin Yvon spectrometer model T64000. In order to avoid sample damage or structural transitions due to the electromagnetic radiation incidence, mainly in the UV range, the incident power was kept always under $0.50 \mathrm{~mW}$ with a spot diameter of approximately $2 \mu \mathrm{m}$. With these parameters the power density was kept always under $625 \mathrm{~mW} / \mathrm{mm}^{2}$, many orders of magnitude below $1500 \mathrm{~W} / \mathrm{mm}^{2}$, which is the annealing threshold for a-C:H films. In any case, a number of measurements were carried out in the same point with different time exposure, beginning from short time to longer ones, to verify the reproducibility of the experiments. After certifying that the experimental conditions did not alter the samples physical properties, Raman spectra were collected for different regions of each sample to verify their homogeneities.

Krypton atomic concentrations of the films were determined by RBS. The measurements were carried out using a $\mathrm{He}^{+}$beam with energy of $2.2 \mathrm{MeV}$. The samples thickness was in the range of 700-760 $\mathrm{nm}$, determined with a profilometer scanner. The intrinsic stress of the films was obtained with the modified Stoney's equation using the substrate curvature before the deposition and the curvature of the film/ substrate system after deposition, determined by means of the bending beam method, described in details elsewhere. ${ }^{42}$

\section{RESULTS AND DISCUSSIONS}

\section{A. $\mathrm{Kr} / \mathrm{C}$ composition}

The incorporation of krypton atoms in the a-C:H films prepared in an atmosphere composed by a mixture of methane $(70 \%)$ and krypton $(30 \%)$ gases depends strongly on the krypton ion energy [Fig. 1(a)]. The krypton content initially increases, from 0 to 0.9 at. $\%$, in the 0 to approximately $-150 \mathrm{~V}$ range, and then decreases for the rest of ion energies 
(proportional to the bias voltage) investigated in this work. This behavior is very similar to that obtained with Xe introduced into amorphous silicon ${ }^{43,44}$ deposited by the IBAD technique, in which $\mathrm{Xe}$ atoms are introduced into the film using an ion gun to assist the deposition. A similar phenomenon has been reported in tetrahedral amorphous carbon (ta-C) deposited by FCVA, considering the concentration of $s p^{3}$ hybridization as a function of $\mathrm{C}^{+}$ion energy. ${ }^{14,21,34}$ The existence of a maximum of $s p^{3}$ at approximately the same energy $(100 \mathrm{eV}-200 \mathrm{eV})$ has been explained in terms of the subplantation model. ${ }^{14,34-37}$ In this model, the content of incident carbon atoms implanted into the film increases rapidly with carbon energy (up to about $100 \mathrm{eV}$ ), promoting the incorporation of carbon atoms in the metastable $s p^{3}$ configuration due to the densification. For ions with high energy (above the range of about 100-150 eV) part of the energy is transformed into heat, through the thermal spike effect, which relaxes the $s p^{3}$ hybridization back to $s p^{2}$, as a consequence of the carbon atoms diffusion to the film surface, as proposed by Robertson ${ }^{35}$ and Davis. ${ }^{37}$ One can also use the subplantation model to explain the result reported in Fig. 1(a) considering that the film is bombarded by krypton ions, instead of carbon ions. At low energy, the content of krypton ions incorporated into the films increases with krypton ion energy. When the ion energy is high (above the threshold energy for the incorporation of krypton) the thermal spike effect promotes relaxation of the carbon network, resulting in partial effusion of the incorporated krypton atoms. Thus, the concentration of krypton in the films starts to reduce when the thermal spike effect becomes intense [above $100 \mathrm{eV}$ in Fig. 1(a)].

Figure 1(b) displays the concentration of $\mathrm{Kr}$ as a function of the $\mathrm{Kr}$ partial pressure. In this series of films we choose a bias voltage of $-120 \mathrm{~V}$, which is the one that leads to the highest incorporation of krypton atoms in an atmosphere of Kr partial pressure of $30 \%$ [Fig. 1(a)]. As it can be observed in Fig. 1(b), the amount of krypton atoms trapped within the amorphous matrix increases monotonically with the increase of the krypton partial pressure in the range studied, reaching values up to 1.6 at. $\%$ for a partial pressure of $50 \%$.

\section{B. Structural properties}

\section{Visible and ultraviolet Raman of a-C:H with krypton as a function of bias voltage}

The way $s p^{2}$ atoms are distributed within the amorphous carbon matrix can be better analyzed by means of Raman scattering spectroscopy in the visible [Fig. 2(a)] and UV [Fig. 2(b)] ranges. For low self bias, the visible Raman spectra [Fig. 2(a)] are characteristic of DLC structures. ${ }^{4}$ As the deposition energy increases the $\mathrm{D}$ peak rises, indicating an increase in number and size of graphitic clusters.

Figure 3 displays important parameters extracted from the Raman spectra such as $G$ peak position $[\operatorname{Pos}(G)], I(D) /$ $\mathrm{I}(\mathrm{G})$ ratio, $\mathrm{G}$ peak full width at half maximum (FWHM) and $\mathrm{G}$ peak dispersion [defined as $\operatorname{Disp}(\mathrm{G})=\left[\operatorname{Pos}(\mathrm{G})_{\mathrm{UV}}\right.$ $\left.\left.-\operatorname{POS}(\mathrm{G})_{\mathrm{Vis}}\right] /\left[\lambda_{\mathrm{Vis}}-\lambda_{\mathrm{UV}}\right]\right]$. To obtain these parameters the spectra were fitted using two Gaussians. The $\mathrm{I}(\mathrm{D}) / \mathrm{I}(\mathrm{G})$ ratio
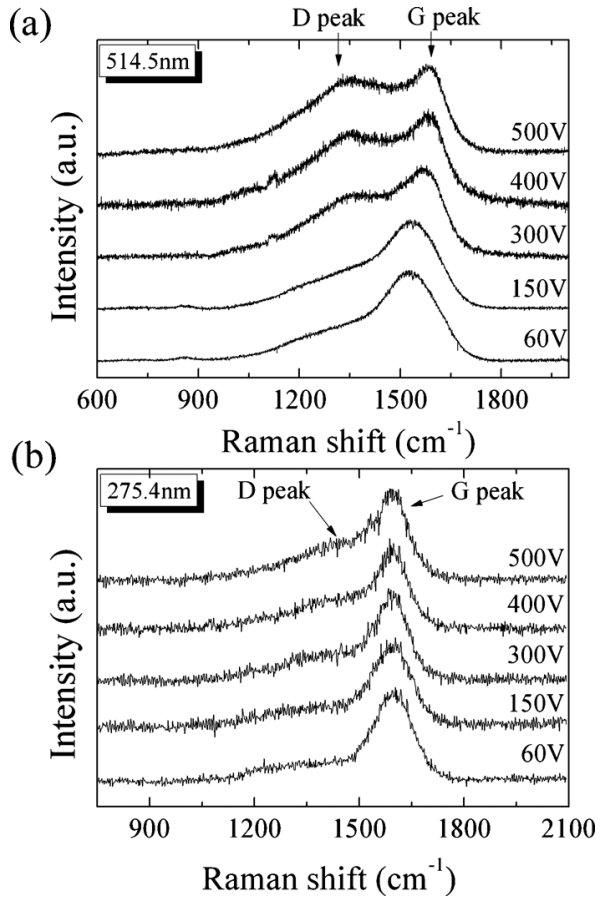

FIG. 2. Raman spectra obtained from $\mathrm{Kr}$ free samples deposited with different self-bias voltages with excitation radiation in the visible (a) and in the UV range (b).

[Fig. 3(a)] increases from 0.3 to 1.4 in the visible Raman and from 0.25 to 0.6 in the UV Raman, for both series of a-C:H with $\mathrm{Kr}$ incorporated and $\mathrm{Kr}$ free samples. The increase in the $\mathrm{I}(\mathrm{D}) / \mathrm{I}(\mathrm{G})$ ratio of amorphous carbon has been associated with an increase in the number and size of $s p^{2}$ hybridized closed structures.

$\operatorname{Pos}(\mathrm{G})$ shifts from approximately $1535-1585 \mathrm{~cm}^{-1}$ as a function of the self bias for the visible Raman. In the case of

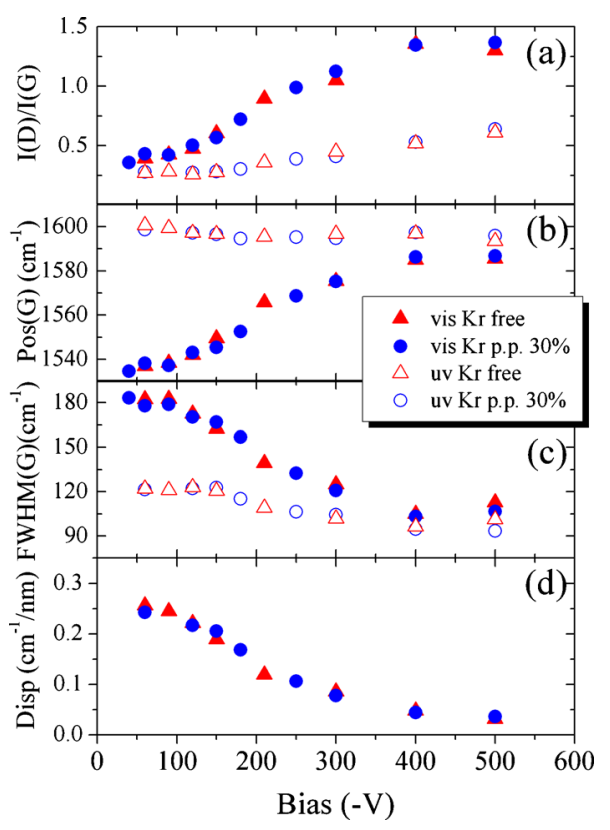

FIG. 3. (Color online) Parameters extracted from visible and UV Raman: (a) $\mathrm{I}(\mathrm{d}) / \mathrm{I}(\mathrm{g})$, (b) G peak position (c) G peak FWHM and (d) G peak dispersion, as a function of deposition bias voltage of $\mathrm{Kr}$ free and $\mathrm{Kr}$ incorporated a-C:H films. 
UV Raman the Pos $(\mathrm{G})$ is almost insensitive to the bias voltage. A shift to higher wave numbers can be related to an increase in $s p^{3}$ atomic fraction or to an increase in structural order. $^{4,5,45}$ It is known ${ }^{4,5,45}$ that $\operatorname{Pos}(\mathrm{G})$ obtained by UV Raman is much more sensitive to the $s p^{3}$ concentration than to the structural order, while the opposite is observed in the visible Raman. Thus, based on this results one can conclude that the $\operatorname{Pos}(\mathrm{G})$ shift displayed in Fig. 3(b) is associated with $s p^{2}$ atoms ordering. In other words, increasing the bias voltage we observe an increase in structural order, from a structure composed mainly by isolated $s p^{2}$ bonds (typical of DLC) to a structure composed of a large number of clusters of aromatic rings, with a consequent reduction in the number of $s p^{3} \mathrm{C}-\mathrm{C}$ bonds (typical of GLC). A better way to analyze this two peaks (visible and UV) is to observe the behavior of the G peak dispersion [Disp(G)] [Fig. 3(d)]. It clearly decreases with bias voltage. The same behavior is also observed for FWHM(G) [Fig. 3(c)]. For a-C:H the FWHM $(\mathrm{G})$ is related to the structural disorder (angles and length of the bonds) while the $G$ peak dispersion $[\operatorname{Disp}(G)]$ is related to the topological disorder (variations in the size and shape of $s p^{2}$ clusters). Thus, the FWHM(G) showed in Fig. 3(c) and the Dips(G) in the Fig. 3(d) indicate that the fraction of $s p^{2}$ atoms increases and gets rearranged in more organized structures for high bias voltages.

All the parameters obtained by Raman in the visible and UV region shown in Fig. 3 are basically the same for samples with (circles) and without (triangles) krypton into the carbon matrix, in spite of the variation in the krypton concentration in the entire range. These results reinforce the previous interpretation that the structural changes observed in Fig. 3 are caused by the energy of the hydrocarbon ions, from the plasma dissociation of methane molecules, and not caused by the incorporation of $\mathrm{Kr}$ atoms into the matrix.

\section{Visible and ultraviolet Raman of a-C:H as a function of krypton partial pressure}

The parameters extracted from the Raman spectra with radiation in the visible and UV ranges obtained for the series of samples prepared as a function of the Kr p.p are showed in Fig. 4. As observed in Fig. 1(b), the incorporation of $\mathrm{Kr}$ increases monotonically from zero to 1.6 at. $\%$ in the $0 \%-$ $50 \%$ range of $\mathrm{Kr}$ p.p. From the increase in the $\mathrm{I}(\mathrm{D} /) \mathrm{I}(\mathrm{G})$ ratio, mainly in the range of $5 \%$ and $20 \%$ of $\mathrm{Kr}$ [Fig. 4(a)], one can argue that there is a small increase in the concentration of graphitic structures as the partial pressure of $\mathrm{Kr}$ atoms in the atmosphere raises. There is also a small variation in the $\operatorname{FWHM}(\mathrm{G})$ in the visible range, indicating an structural order improvement, but the $G$ peak position stays almost constant. The dispersion, showed in Fig. 4(d), is in agreement with the FWHM $(\mathrm{G})$ result, having the same behavior. In summary, the increase in the $\mathrm{Kr}$ partial pressure, resulting in the incorporation of up to 1.6 at. \% of $\mathrm{Kr}$ atoms into the carbon matrix, does not change the structure of the film significantly. Comparing the variations in the Raman parameters from films deposited as a function of bias voltage, with and without $\mathrm{Kr}$ (Fig. 3) and as a function of $\mathrm{Kr}$ partial pressure

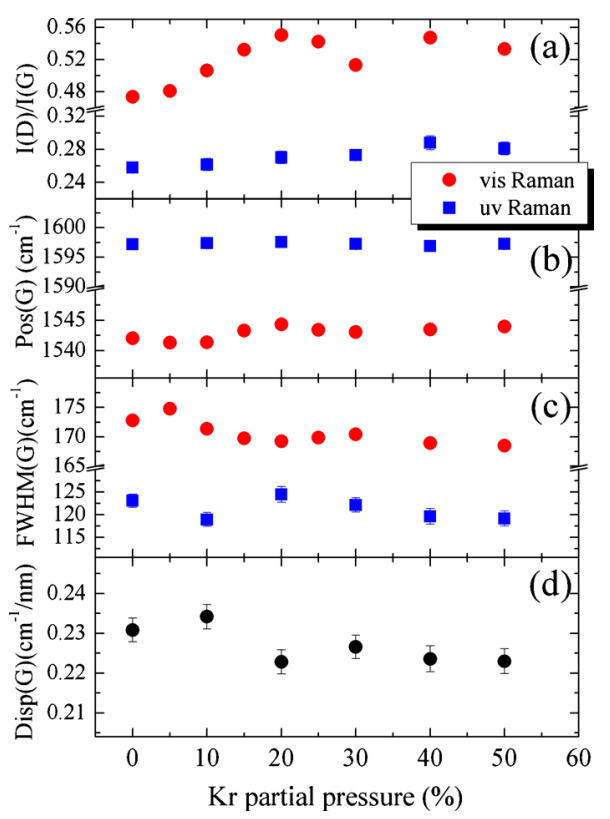

FIG. 4. (Color online) Parameters extracted from visible and UV Raman: (a) $\mathrm{I}(\mathrm{d}) / \mathrm{I}(\mathrm{g})$, (b) G peak position and (c) G peak FWHM and (d) G peak dispersion, as a function of $\mathrm{Kr}$ partial pressure

(Fig. 4) one verifies that the structural changes induced by the incorporation of $\mathrm{Kr}$ are very small compared to that induced by varying the bias voltage.

The small increase in the structural order of the $s p^{2}$ bonds due to the incorporation of krypton, observed in Fig. 4 , can be understood as a consequence of the incorporation process of krypton atoms. When a $\mathrm{Kr}$ ion penetrates through the growing film surface it loses a fraction of its energy, as thermal energy, to the neighbor carbon atoms. This energy could be sufficient to induce a $s p^{3} \rightarrow s p^{2}$ transition, as the $s p^{3}$ hybridization is a metastable state. On the other hand, since the $\mathrm{Kr}$ atom is 1.5 times bigger than the carbon atom, its presence could diminish the volume available within the film for the carbon atoms, which could induces $s p^{2} \rightarrow s p^{3}$ transitions, similarly to the formation of $s p^{3}$ bonds explained by the subplantation model. ${ }^{14,34-36}$ However, krypton atoms do not establish chemical bonds with $\mathrm{C}$ atoms. Thus, the $\mathrm{Kr}$ atom can move inside the matrix and reach a low energy state, which is mainly inside a void. Consequently, what changes the matrix structure of the films is not the presence of $\mathrm{Kr}$ atoms, but the interaction between the penetrating $\mathrm{Kr}$ atoms and the host matrix carbon atoms.

\section{FTIR of a-C:H with krypton}

Hydrogen concentration has been frequently determined using infrared spectroscopy. For Si and Ge the infrared absorption bands related to $\mathrm{Si}-\mathrm{H}$ and $\mathrm{Ge}-\mathrm{H}$ bonds are well defined structures even in amorphous matrices, such that the determination of hydrogen concentration has been extensively performed. However, in amorphous carbon, the IR absorption bands related to $\mathrm{C}-\mathrm{H}$ bonds have much more complex structures, compared with $\mathrm{Si}$ and $\mathrm{Ge}$, due to the different types of carbon hybridization and bond configuration, like $s p^{3} \mathrm{CH}, s p^{3} \mathrm{CH}_{2}, s p^{3} \mathrm{CH}_{3}$, aromatic $s p^{2} \mathrm{CH}$, aliphatic $s p^{2} \mathrm{CH}$, and $s p^{2} \mathrm{CH}_{2}$. These different bond configu- 


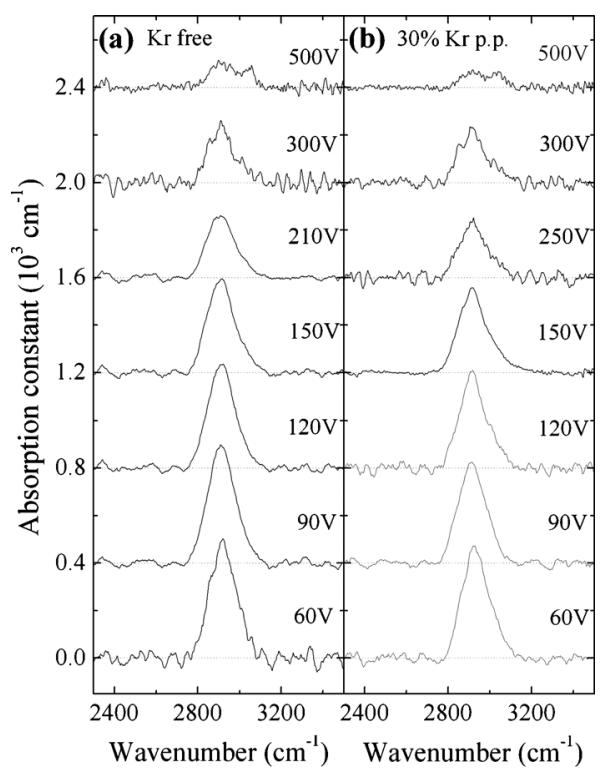

FIG. 5. Carbon-hydrogen stretching vibrational mode for $\mathrm{Kr}$ free (a) and $\mathrm{Kr}$ incorporated a-C:H films deposited by PECVD, as a function of the bias voltage.

rations in carbon structures change the interaction energy between carbon and hydrogen atoms, which determines the vibrational mode frequencies. ${ }^{41,46}$ A lot of work had been done to investigate the $\mathrm{C}-\mathrm{H}$ vibrational modes in organic molecules to understand and determine the characteristic frequency of each type of bonding configuration. Today, these values are well known and can be found in a number of handbooks. ${ }^{47-51}$ Ristein et al. ${ }^{41}$ had described, in a very complete way, the relations between $\mathrm{C}-\mathrm{H}$ vibrational mode frequency in organic molecules and that in amorphous $\mathrm{C}-\mathrm{H}$ matrix, mainly for the stretching modes.

In Fig. 5 we compare the evolution of the $\mathrm{C}-\mathrm{H}$ stretching mode (in the $2750-3150 \mathrm{~cm}^{-1}$ range), obtained by FTIR, for pure a-C:H and for $\mathrm{Kr}$ incorporated samples as a function of bias voltage. Increasing the bias voltage, a reduction on the hydrogen concentration (proportional to the band area) can be observed for values higher than $-150 \mathrm{~V}$. There is not much difference between the films prepared with or without $\mathrm{Kr}$ in the atmosphere. A roughly featureless band is observed in the entire range of bias investigated, which is characteristic of DLC and GLC materials, in contrast with PLC films with well defined bands associated with a large variety of possible $\mathrm{C}-\mathrm{H}$ configurations. In the present data (Fig. 5), it is not possible to distinguish the different contributions from different $\mathrm{C}-\mathrm{H}$ bond configurations, as it is possible in the case of PLC films. The bending $\mathrm{C}-\mathrm{H}$ mode, which could bring more information, is not presented here since it is usually mixed with other contributions, such as $\mathrm{C}=\mathrm{C}$ bonds, which makes its analysis even more complicated.

For the films deposited with different $\mathrm{Kr}$ partial pressure (Fig. 6), even though the stretching bands look very similar, it is possible to notice an asymmetry in the peak for high $\mathrm{Kr}$ percentages. For these samples the stretching peak extends to higher values of wave numbers, superior to $3000 \mathrm{~cm}^{-1}$. At about $3050 \mathrm{~cm}^{-1}$ the absorption is related to aromatic $s p^{2}$

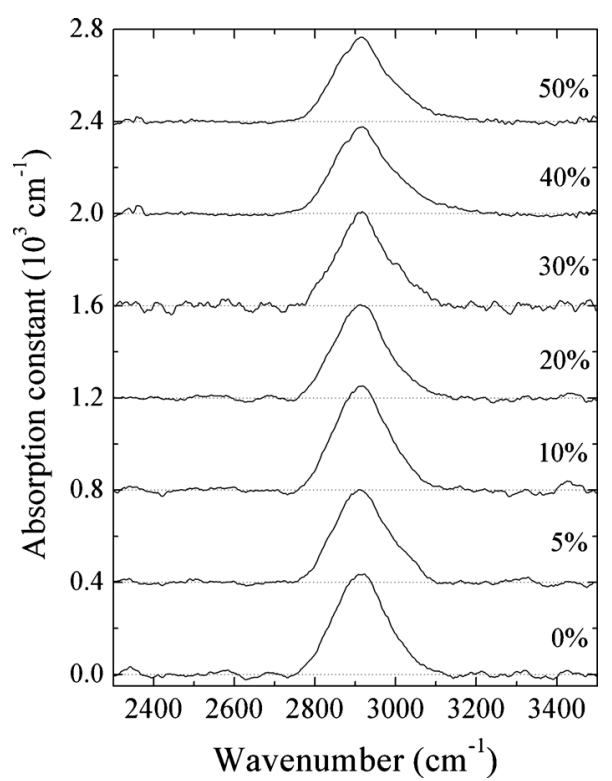

FIG. 6. Carbon-hydrogen stretching vibrational mode of films deposited with different $\mathrm{CH} 4 / \mathrm{Kr}$ atmosphere compositions.

$\mathrm{CH}^{41}$ Thus, the absorption enhancement in this region indicates an increase in the quantity of this type of structural configuration, which means that the films became slightly more graphitic for increasing $\mathrm{Kr}$ pressure. This result is consistent with the small increase in the $\mathrm{I}(\mathrm{D}) / \mathrm{I}(\mathrm{G})$ ratio for both UV and Visible Raman for increasing $\mathrm{Kr}$ pressure accompanied by a small decrease in the $\operatorname{FWHM}(G)$ in the visible range (Fig. 4).

Ristein et al. $^{41}$ showed that it is possible to reasonably estimate the $\mathrm{H}$ content in a-C:H films by calculating the mean absorption cross section of different types of $\mathrm{C}-\mathrm{H}$ configuration applying the following relation:

$$
N_{H}=\frac{\int \alpha(v) d v}{\langle v\rangle_{\sigma}}
$$

where $\alpha$ is the absorption coefficient, $v$ is the wave number; $\langle v\rangle$ is the peak mean wave number, and $\sigma$ the absorption cross-section. For non-PLC materials the calculated cross section is given by $\sigma=8 \times 10^{-22} \mathrm{~cm}^{2}$, while for PLC is $\sigma$ $=1.6 \times 10^{-21} \mathrm{~cm}^{2}$. As the samples studied in this work showed properties of DLC and GLC films, we applied Eq. (1) with $\sigma=8 \times 10^{-22} \mathrm{~cm}^{2}$ to the spectra of Figs. 5 and 6 . The results obtained are shown in Fig. 7(a) for the samples deposited with different self-bias and (b) for different $\mathrm{Kr} / \mathrm{CH}_{4}$ compositions. For samples prepared with different $\mathrm{Kr} / \mathrm{CH}_{4}$ compositions, the estimated hydrogen concentration is independent of the growth condition, being of about $(23 \pm 2) \%$ for all samples in this series. For a-C:H, almost all of the $s p^{3}$ sites are hydrogenated, while the same is not true for the $s p^{2}$ ones. ${ }^{19,52}$ As a consequence, the $\mathrm{H}$ content is related to $s p^{3}$ bond fraction inside the material. Thus, the $\mathrm{H}$ content reduction from $\sim 25 \%$ to $\sim 6 \%$ is mainly related to the decrease in $s p^{3}$ atoms concentration, characterizing a transition from DLC to GLC structures for increasing deposition energy, as it was also observed by Raman (Sec. III B 1). On the other hand, it was not observed any variation in $\mathrm{H}$ concentration, within experimental error, for samples 


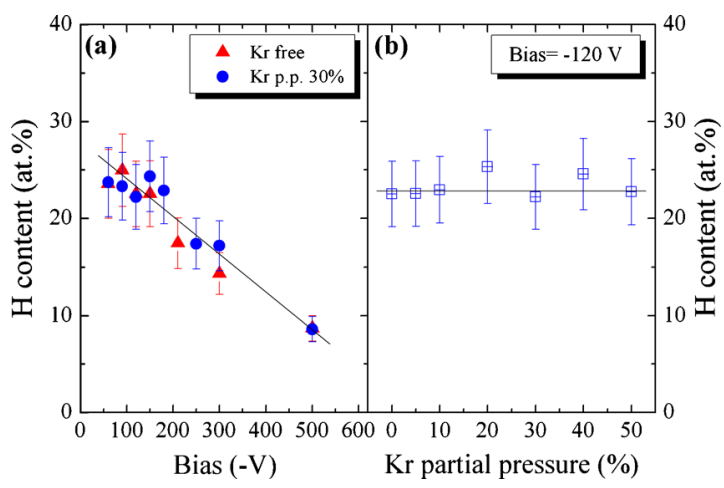

FIG. 7. (Color online) Hydrogen content of a-C:H films, deposited with and without krypton in the atmosphere, estimated from FTIR data using the approximation proposed by Ristein et al. (Ref. 41) for films deposited as function of the bias voltages (a) and $\mathrm{Kr}$ partial pressure (b).

deposited with different Kr pressure [Fig. 7(b)]. Even though it was observed an increase in $s p^{2}$ composition and ordering with increasing $\mathrm{Kr}$ pressure, it was shown that this effect is too small compared to that induced by the bias voltage. As the $\mathrm{H}$ content is an approximation, any variation in the actual $\mathrm{H}$ content could hardly be determined using IR. One should remember that the asymmetry that arises in the stretching vibrational mode peak with increasing $\mathrm{Kr}$ partial pressure (Fig. 6) is due to the appearance of $s p^{2}$ aromatic structure, which has a different absorption cross section that is not considered in the approximation adopted to determine the concentration of hydrogen, $N_{H}$.

\section{Optical properties}

The optical band gap, $E_{04}$, of a-C:H structures deposited with and without $\mathrm{Kr}$ is displayed in Fig. 8(a) for samples deposited as a function of the bias voltage, and in Fig. 8(b) for samples deposited as a function of $\mathrm{Kr}$ partial pressure. The decrease in $E_{04}$ with increasing bias voltage is related to an increase in the number of $s p^{2}$ atoms and in the $s p^{2}$ sites structural order, as was observed by FTIR and Raman. These effects are well known for pure a-C:H deposited by PECVD. Samples deposited with bias voltages higher than $-400 \mathrm{~V}$ have some decimals of electron volts of optical gap, showing that they have a high concentration of $s p^{2}$ clusters organized

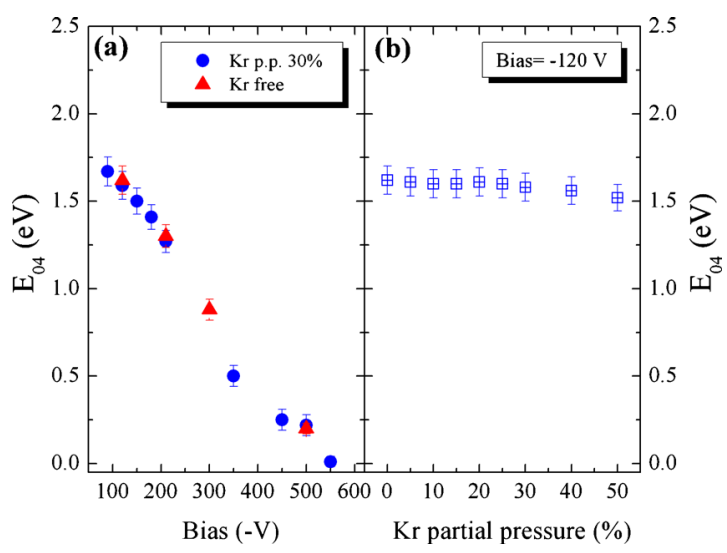

FIG. 8. (Color online) Comparison between the dependence of the optical gap $E_{04}$ on the bias voltage for $\mathrm{Kr}$ free and $\mathrm{Kr}$ incorporated a-C:H films (a) and on the $\mathrm{Kr}$ partial pressure (b).

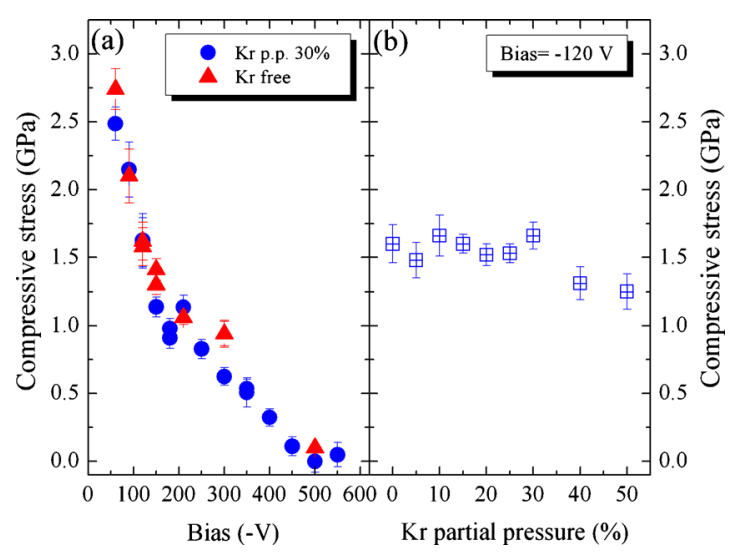

FIG. 9. (Color online) (a) Compressive stress as a function of the bias voltage for $\mathrm{Kr}$ free and $\mathrm{Kr}$ incorporated a-C:H films. (b) Compressive stress of a-C:H as a function of $\mathrm{Kr}$ partial pressure in the deposition atmosphere.

in aromatic rings, according to the model proposed by Robertson. ${ }^{1,5,52}$ Figure 8(b) shows that the band gap decreases slightly as the $\mathrm{Kr}$ partial pressure changes from zero to $50 \%$, which correspond to an increase in the concentration of $\mathrm{Kr}$ in the sample from zero to 1.6 at. \%. The effect of introducing $\mathrm{Kr}$ in the matrix does not affect much the band gap of the carbon structures. The slight decrease in this parameter is related to the small increase in $s p^{2}$ sites, as revealed by Raman scattering spectroscopy.

\section{Mechanical properties}

In Fig. 9(a) it is shown the compressive stress for samples prepared as a function of bias voltage and in Fig. 9(b) for samples deposited as a function of krypton partial pressure. A drastic reduction of the stress, from approximately $2.7 \mathrm{GPa}$ to about zero, is observed as the bias voltage varies from zero to $-550 \mathrm{~V}$. As can be observed in Fig. 9(a), there is no difference in the stress when we introduce krypton in the films in the range of bias voltage investigated. For films deposited at $-120 \mathrm{~V}$, but varying the krypton pressure, Fig. 9(b), a slight decrease in the stress is observed for krypton dilution higher than $30 \%$. These results show that the mechanical properties of those structures are much more sensitive to the deposition energy than to the $\mathrm{CH}_{4} / \mathrm{Kr}$ atmosphere composition. They also indicate that the structures of the films are not much influenced by the incorporation of krypton up to about 1.6 at. \%. These results are in agreement with those obtained by Raman and FTIR.

The high values of mechanical stress, up to $2.7 \mathrm{GPa}$, may be related to the concentration of $s p^{3}$ bonds and to the structural disorder. ${ }^{53}$ The decrease in the compressive stress with bias may be related to a reduction in the number of $s p^{3}$ hybridized carbon atoms as well as to an increase in the structural order, as revealed by Raman. Since the mechanical stress basically vanishes for high bias, it indicates that those structures are in a relaxed state, with few distorted bonds and sites, in a lower energy configuration. This explanation is consistent with the results of Raman observed in Sec. III B, which indicates that those structures are well ordered, independently on the presence of $\mathrm{Kr}$ during the deposition. 
The behavior displayed in Fig. 9 is completely different from those reported for amorphous carbon deposited by IBAD using a secondary beam of noble gases $(\mathrm{Ar}, \mathrm{Kr}$, and $\mathrm{Xe})$ to simultaneously bombard the film during deposition. ${ }^{17,31}$ The mechanical stress of those films increases substantially (up to about $10 \mathrm{GPa}$ ) with the assisting noble gas energy in the same range of energy investigated in this work. This difference may be related to the difference in the plasma dynamics between the IBAD and PECVD techniques. First, the samples reported in Refs. 17 and 31 are nonhydrogenated, while films prepared in this work, by PECVD, are hydrogenated. Second, ion beam generated by an ion gun has a sharp velocity distribution due to the ion acceleration process, which is performed by a dc voltage power supply. In contrast, the ion energy of noble gases generated by rf-PECVD is distributed in a broader range of energy due to the nature of a sinusoidal oscillation of the 13.56 $\mathrm{MHz}$ power supply. For IBAD prepared samples the $\mathrm{C}^{+}$ion energy was kept constant while the noble gases ionic beam energy was varied. In this work the carbon precursors had their energy varied with the $\mathrm{Kr}$ ions energy, as it was controlled by a self-bias applied to the substrate.

It is intriguing that the concentration of Krypton (Fig. 1) resembles the behavior obtained by IBAD, while the stress follows a completely different behavior. However, opposite to the IBAD prepared carbon, ${ }^{17,31}$ no significant difference between structures grown with or without krypton was observed. It seems that the film structure is mainly defined by the carbon growth kinetics, and not by the energy and amount of krypton ions in the deposition atmosphere. Even thought the maximum of $s p^{3}$ bonds (based on Raman, FTIR, optical and mechanical characterization) looks to occur for deposition energies of about $50 \mathrm{eV}$, the growth process can be still described by the subplantation model. Applying dynamic Monte Carlo simulations to the synthesis of a-C:H using $\mathrm{CH}_{3}$ as carbon precursors, Miyagawa et al. ${ }^{54}$ were able to describe the a-C:H growth mechanism based on the subplantation model, obtaining results with a quite good agreement with experiments. They had shown that the amount of $s p^{3}$ carbon atoms in the final structure is also dependent on the $\mathrm{CH}_{3}^{+} / \mathrm{CH}_{3}$ ratio in the deposition atmosphere, where high $\mathrm{CH}_{3}^{+} / \mathrm{CH}_{3}$ ratio shifts the $s p^{3}$ maximum to lower deposition energies. As the rf-power changes with the self-bias applied to the substrates, films prepared with different energy are also prepared with plasmas with different proportions of $\mathrm{CH}_{3}$ and $\mathrm{CH}_{3}^{+}$, what can explain the observed dependence of the films properties with the deposition energy. On the other hand, the $\mathrm{Kr}$ incorporation depends mainly on the $\mathrm{Kr}$ ions energy and partial pressure, being similar to the incorporation of noble gases by IBAD.

\section{CONCLUSIONS}

In this work a-C:H structures were grown by PECVD using $\mathrm{CH}_{4} / \mathrm{Kr}$ atmosphere. The concentration of $\mathrm{Kr}$, in the $0-1.6$ at. \% range, increases monotonically with $\mathrm{Kr}$ partial pressure and is more efficient in the -100 up to $-200 \mathrm{~V}$ bias voltage range, which is the range in which the concentration of $s p^{3} \mathrm{C}-\mathrm{C}$ sites and hardness are higher, according to the subplantation model. The dependence of $\mathrm{Kr}$ incorporation as a function of bias voltage was explained in terms of the subplantation model, which has been used to explain the maximum observed in $s p^{3}$ hybridization in amorphous carbon films prepared by FCVA. Using Raman scattering spectroscopy with excitation radiation in the visible and UV range, FTIR, optical properties and mechanical stress it was observed that the presence of krypton in the deposition atmosphere makes small difference in the film properties. These results indicate that the film structure is determined by the carbon growth kinetics, which depends more on the energy and on the ion/neutral ratio of hydrocarbons radicals than on the krypton energy or partial pressure, in contrast with data reported for films deposited by IBAD using an ion gun to simultaneously bombard the films with noble gases during deposition.

\section{ACKNOWLEDGMENTS}

This work was supported by FAPESP, CAPES, MCT, and $\mathrm{CNPq}$ finance agencies as well as by the Spanish Ministry of Science and Innovation for financial support through Grant No. MAT2009-10350. The authors are in debt to CCS/ UNICAMP and LAMFI/USP for FTIR and RBS measurements, respectively.

${ }^{1}$ J. Robertson and E. P. O'Reilly, Phys. Rev. B 35, 2946 (1987).

${ }^{2}$ J. C. Angus and Y. Wang, in Diamond and Diamond-Like Films and Coatings, edited by R. E. Clausing (Plenum, New York, 1991).

${ }^{3}$ P. C. Kelires, Phys. Rev. B 47, 1829 (1993).

${ }^{4}$ C. Casiraghi, A. C. Ferrari, and J. Robertson, Phys. Rev. B 72, 085401 (2005).

${ }^{5}$ A. C. Ferrari and J. Robertson, Phys. Rev. B 61, 14095 (2000).

${ }^{6}$ F. Piazza and G. Morell, Diamond Relat. Mater. 18, 43 (2009).

${ }^{7}$ Y. Lifshitz, G. D. Lempert, E. Grossman, H. J. Scheibe, S. Voellmar, B. Schultrich, A. Breskin, R. Chechik, E. Shefer, D. Bacon, R. Kalish, and A. Hoffman, Diamond Relat. Mater. 6, 687 (1997).

${ }^{8}$ A. Champi, R. G. Lacerda, G. A. Viana, and F. C. Marques, J. Non-Cryst. Solids 338-340, 499 (2004).

${ }^{9}$ F. C. Marques, R. G. Lacerda, A. Champi, V. Stolojan, D. C. Cox, and S. R. P. Silva, Appl. Phys. Lett. 83, 3099 (2003).

${ }^{10}$ S. Prawer, K. W. Nugent, Y. Lifshitz, G. D. Lempert, E. Grossman, J. Kulik, I. Avigal, and R. Kalish, Diamond Relat. Mater. 5, 433 (1996).

${ }^{11}$ M. H. Oliveira, Jr., D. S. Silva, A. D. S. Côrtes, M. A. B. Namani, and F. C. Marques, Diamond Relat. Mater. 18, 1028 (2009).

${ }^{12}$ V. G. Litovchenko and N. I. Klyui, Sol. Energy Mater. Sol. Cells 68, 55 (2001).

${ }^{13}$ M. Hakovirta, Diamond Relat. Mater. 8, 1225 (1999).

${ }^{14}$ J. Robertson, Mater. Sci. Eng. R. 37, 129 (2002).

${ }^{15}$ K. R. Roy and K. R. Lee, J. Biomed. Mater. Res., Part B: Appl. Biomater. 83B, 72 (2007).

${ }^{16}$ A. C. Ferrari, Surf. Coat. Technol. 180-181, 190 (2004).

${ }^{17}$ R. G. Lacerda, P. Hammer, C. M. Lepienski, F. Alvarez, and F. C. Marques, J. Vac. Sci. Technol. A 19, 971 (2001).

${ }^{18}$ P. Koidl, Ch. Wild, B. Discheler, J. Wagner, and M. Ramsteiner, Mater. Sci. Forum 52-53, 41 (1990).

${ }^{19}$ M. A. Tamor, W. C. Vassell, and K. R. Carduner, Appl. Phys. Lett. 58, 592 (1991).

${ }^{20}$ P. J. Fallon, V. S. Veerasamy, C. A. Davis, J. Robertson, G. A. J. Amaratunga, W. I. Milne, and J. Koskinen, Phys. Rev. B 48, 4777 (1993).

${ }^{21}$ M. Chhowalla, J. Robertson, C. W. Chen, S. R. P. Silva, C. A. Davis, G. A. J. Amaratunga, and W. I. Milne, J. Appl. Phys. 81, 139 (1997).

${ }^{22}$ D. R. McKenzie, D. Muller, and B. A. Pailthorpe, Phys. Rev. Lett. 67, 773 (1991).

${ }^{23}$ R. Lossy, D. L. Pappas, P. A. Roy, J. J. Cuomo, and V. M. Sura, Appl. Phys. Lett. 61, 171 (1992).

${ }^{24}$ A. Anders, S. Anders, I. G. Brown, M. R. Dickinson, and R. A. MacGill, J. Vac. Sci. Technol. B 12, 815 (1994). 
${ }^{25}$ Y. Lifshitz, S. R. Kasi, and J. W. Rabalais, Phys. Rev. Lett. 62, 1290 (1989).

${ }^{26}$ D. L. Pappas, K. L. Saenger, J. Brueley, W. Krakow, J. J. Cuomo, T. Gu, and R. W. Collins, J. Appl. Phys. 71, 5675 (1992).

${ }^{27}$ F. Xiong, Y. Y. Chang, and R. P. H. Chang, Phys. Rev. B 48, 8016 (1993).

${ }^{28}$ A. A. Voevodin, S. J. P. Laube, S. W. Walck, J. S. Solomon, M. S. Donley, and J. S. Zabinski, J. Appl. Phys. 78, 4123 (1995).

${ }^{29}$ J.-I. Matsuda and T. Maekawa, Geochem. J. 26, 251 (1992).

${ }^{30}$ K. Fukunaga and J.-I. Matsuda, Geochem. J. 31, 263 (1997)

${ }^{31}$ R. G. Lacerda, M. C. dos Santos, L. R. Tessler, P. Hammer, F. Alvarez, and F. C. Marques, Phys. Rev. B 68, 054104 (2003).

${ }^{32}$ L. K. Cheah, X. Shi, B. K. Tay, and E. Liu, Surf. Coat. Technol. 105, 91 (1998).

${ }^{33}$ T.-Y. Kim, C. S. Lee, Y. J. Lee, K. H. Chae, and K. H. Oh, J. Appl. Phys. 101, 023504 (2007).

${ }^{34}$ J. Robertson, Diamond Relat. Mater. 14, 942 (2005).

${ }^{35}$ J. Robertson, Diamond Relat. Mater. 2, 984 (1993).

${ }^{36}$ J. Robertson, Diamond Relat. Mater. 3, 361 (1994).

${ }^{37}$ C. A. Davis, Thin Solid Films 226, 30 (1993).

${ }^{38}$ I. Chambouleyron, J. M. Martínez, A. C. Moretti, and M. Mulato, Appl. Opt. 36, 8238 (1997).

${ }^{39}$ E. G. Birgin, I. Chambouleyron, and J. M. Martínez, J. Comput. Phys. 151, 862 (1999).

${ }^{40}$ I. Chambouleyron, S. D. Ventura, E. G. Birgin, and J. M. Martínez, J. Appl. Phys. 92, 3093 (2002).

${ }^{41}$ J. Ristein, R. T. Stief, L. Ley, and W. Beyer, J. Appl. Phys. 84, 3836 (1998).
${ }^{42}$ M. M. de Lima, Jr., R. G. Lacerda, J. Vilcarromero, and F. C. Marques, J. Appl. Phys. 86, 4936 (1999).

${ }^{43}$ P. F. Barbieri, M. H. de Oliveira, Jr., A. Champi, and F. C. Marques, J. Non-Cryst. Solids 352, 1016 (2006).

${ }^{44}$ P. F. Barbieri, R. Landers, M. H. de Oliveira, Jr., F. Alvarez, and F. C. Marques, J. Electron Spectrosc. Relat. Phenom. 156-158, 409 (2007).

${ }^{45}$ A. C. Ferrari, A. LiBassi, B. K. Tanner, V. Stolojan, J. Yuan, L. M. Brown, S. E. Rodil, B. Kleinsorge, and J. Robertson, Phys. Rev. B 62, 11089 (2000).

${ }^{46}$ R. Stief, J. Schäfer, J. Ristein, L. Ley, and W. Beyer, J. Non-Cryst. Solids 198-200, 636 (1996).

${ }^{47}$ C. N. R. Rao, Chemical Applications of Infrared Spectroscopy (Academic, New York, 1963).

${ }^{48}$ H. A. Szymanski, Infrared Band Handbook (Plenum, New York, 1963).

${ }^{49}$ G. Herzberg, Molecular Spectra and Molecular Structure (van Nostrand Reinhold, New York, 1945), Vol. II.

${ }^{50}$ L. J. Bellamy, Advances in Infrared Group frequencies (Methuen, London, 1968).

${ }^{51}$ N. B. Colthup, L. H. Daly, and S. E. Wiberley, Introduction to Infrared and Raman Spectroscopy (Academic, New York, 1990).

${ }^{52}$ C. Donnet, J. Fontaine, F. Lefbvre, A. Grill, V. Patel, and C. Jahnes, J. Appl. Phys. 85, 3264 (1999).

${ }^{53}$ A. C. Ferrari, B. Kleinsorge, N. A. Morrison, A. Hart, V. Stolojan, and J. Robertson, J. Appl. Phys. 85, 7191 (1999).

${ }^{54}$ Y. Miyagawa, H. Nakadate, M. Ikeyama, S. Nakao, and S. Miyagawa, Diamond Relat. Mater. 12, 927 (2003). 